\title{
A DisTinçÃo CONTÁvEL-MASSIVO NAS LÍNGUAS NATURAIS*
}

The count-mass distinction in natural languages

\author{
Nize Paraguassu-Martins** \\ Ana Müller**
}

\section{INTRODUÇÃO}

O objetivo deste artigo é investigar a expressão de número e da distinção contável-massivo no sistema nominal das línguas naturais. Em particular, será avaliado o comportamento do nominal nu no Português Brasileiro (PB) com relação às expressões da distinção de número e da distinção contável-massivo. Para tanto, procuraremos responder às seguintes questões: (i) Quais são as denotações possíveis para os nomes comuns (NCs) nas línguas naturais? (ii) Qual seria o papel semântico da morfologia de número e dos classificadores? (ii) O que é ser um nome massivo ou contável?

O artigo defende a hipótese de que a denotação dos NCs contáveis não é necessariamente singular como tradicionalmente assumido, mas pode ser neutra em relação à expressão de número. ${ }^{1}$ Defendemos também que há uma diferença entre denotações neutras e denotações massivas. Por outro lado, como a denotação de um nome contável não é necessariamente singular, a morfologia de número não poderá mais ser considerada como uma operação que transforma singularidades em pluralidades.

O artigo segue a seguinte sequência: na seção 1 , discutimos a distinção contável-massivo e a marcação de número no PB; na seção 2 , discutimos as teorias sobre a relação entre a expressão de número e a

Este trabalho foi apresentado no VI Workshop on Formal Linguistics ocorrido em Florianópolis em 28-29 de agosto de 2006. Agradecemos aos participantes pelos seus comentários.

$$
\text { ** USP }
$$

Entendemos por indivíduos singulares ou atômicos indivíduos cujas partes não configuram indivíduos do mesmo tipo. Assim, nenhuma parte de 'esta mesa' é uma mesa. Já indivíduos plurais são indivíduos compostos de somas de indivíduos singulares do mesmo tipo. Por exemplo, 'estas mesas' consistem de duas ou mais mesas individuais. 
distinção contável-massivo nas línguas naturais, na seção 3, avaliamos essas teorias frente aos dados do PB. Na seção 4, investigamos empiricamente a relação entre a marcação de número e a distinção contável-massivo nas línguas naturais. Finalmente, na seção 5, apresentamos a conclusão.

\section{Nominais Nus, MARCAÇÃo de NÚmERo E Distinção ConTÁvel- MASSIVO NO PB}

Nesta seção, discutimos o comportamento semântico do nominal $\mathrm{nu}$, da marcação de número e da distinção contável-massivo no PB. A maioria das línguas germânicas e românicas permite a ocorrência do plural nu (PN), mas não permite a ocorrência do singular nu (SgN) (cf. CHIERCHIA, 1998, LONGOBARDI, 1994) ${ }^{2}$ O contraste é ilustrado pelas sentenças (1) e (2) do inglês e pelas sentenças (3) e (4) do espanhol.
(1) John bought potatoes.
John comprou batatas
'John comprou batatas'
(2) *John bought potato.
John comprou batata
'John comprou batata'
(3) Juan compró papas.
Juan comprou batatas
'Juan comprou batatas'
(4) *Juan compró papa.
Juan comprou batata
'Juan comprou batata'

Surpreendentemente, podemos observar que o PB permite tanto o SgN como o PN, como ilustram as sentenças em (5a-b) e (6a-b). Nessa língua o uso do SgN (modificado ou não) é extremamente produtivo, não estando sua ocorrência, como em outras línguas, simplesmente ligada aos efeitos eventuais do chamado universal grinder - um 'triturador universal', uma 'máquina' que transforma denotações atômicas contáveis em massa (cf. PELLETIER, 1979).

\footnotetext{
Vamos usar o termo Singular $\mathrm{Nu}$ para nos referirmos ao nominal nu que ocorre sem marca de número e sem determinantes como 'batata' em 'João comprou batata'. o termo é tradicional na literatura sobre o assunto, no entanto, queremos deixar claro que o seu uso não implica que o nominal está marcado para número.
} 
(5) a. João comprou batatas.

b. João comprou batata.

(6) a. Cachorros são inteligentes.

b. Cachorro é inteligente.

Assim como o SgN, no PB, o contraste singular-plural também é produtivo nas expressões nominais e marca uma diferença de significado. Uma expressão nominal como (7a) ou (8a) denota uma única entidade. Já uma expressão nominal como (7b) ou (b) denota duas ou mais entidades. No PB, há dois paradigmas de número. No paradigma padrão, todas as categorias lexicais do sintagma nominal são marcadas para número, como em (7). No paradigma não-padrão, apenas o determinante apresenta marca de número, como em (8). Em ambos o contraste singular-plural é produtivo e significativo.

(7) a. a revista boa.

b. as revistas boas

(8) a.a revista boa.

b. as revista boa

Como observado por Müller (2001) e Schmitt e Munn (1999), NCs e SgNs possuem uma denotação neutra para número no PB. Em (9), o singular nu 'maçã' não apresenta uma semântica de número especificada, sendo compatível tanto com indivíduos singulares quanto com indivíduos plurais. Assim, a sentença (9) é verdadeira tanto em situações em que João comprou apenas uma maçã, como em situações em que ele comprou duas ou mais maçãs.

(9) João comprou maçã.

Nominais indefinidos como o indefinido singular, o SgN e o PN, não apresentam a mesma semântica de número no PB. A sentença (10a) é verdadeira de situações em que João comprou uma (ou mais) maçã(s). Já a sentença (10b) é verdadeira nas situações em que João comprou tanto pedaços/ partes de uma ou mais maçãs como nas situações em que ele comprou uma ou mais maçãs inteiras. ${ }^{3}$ Finalmente, a sentença (10b), com o PN 'maçãs',

Evidentemente, uma sentença como 'João comprou livro ontem', dificilmente seria interpretada como João comprando partes de livros. No entanto, afirmamos que esta é uma possibilidade dada pela língua e que o limite é dado pelo contexto. Um comprador de papel para reciclagem, por exemplo, pode perfeitamente 'comprar livro' por quilo e já desfeitos em partes. 
significa que João comprou duas ou mais maçãs. Assim, vemos que descrições indefinidas e PNs possuem apenas indivíduos em suas denotações. Já NCs e SgNs incluem tanto indivíduos singulares e plurais como partes de indivíduos.

$$
\begin{array}{llll}
\text { (10) a. João } & \text { comprou } & \text { uma } & \text { maçã. } \\
\text { b. João } & \text { comprou } & & \text { maçãa. } \\
\text { c. João } & \text { comprou } & & \text { maçãs. }
\end{array}
$$

Como já observado por Müller 2001, PNs não incluem singularidades em suas denotações. A sentença (11a), com o SgN 'rabo', significa que lagartixa tem um ou mais rabos. Já a sentença (11b), com o PN 'rabos', significa que lagartixa tem dois ou mais rabos (cf. MULLER, 2001).
(11) a. Lagartixa
tem rabo.
b. Lagartixa
têm rabos.

Implementando a análise dos NCs e dos SgNs no PB como possuindo uma denotação neutra em relação à expressão de número singular ou plural, podemos representar essa denotação como em (12). Ou seja, a denotação de NCs contáveis inclui tanto indivíduos singulares quanto suas somas.

(12) $[$ [NCs contáveis] $]=\{a, b, \ldots\{a, b\},\{a, c\}, \ldots,\{a, b, c\} \ldots$, porções de $a, \ldots$, porções de $\{a, b, c\}, \ldots\}$

Ao assumirmos que o NC possui uma denotação neutra em relação ao número, temos de repensar o significado da morfologia de singular e de plural. Tradicionalmente, o plural é analisado como uma operação que cria indivíduos plurais a partir de denotações de NCs que são inerentemente singulares (ver LINK, 1983; CHIERCHIA, 1998). No entanto, se NCs possuem denotações neutras para número, tanto o singular como o plural, em línguas em que estas operações existem, devem ser vistos como operações sobre essas denotações neutras (cf. MÜLLER, 2001). O operador singular seria um operador que seleciona apenas entidades atômicas na denotação do $\mathrm{NC}$, como formalizado em (13a) e ilustrado em (13b). Já o operador plural, seleciona o conjunto de todas as pluralidades na denotação do NC, como formalizado em (14a) e ilustrado em (14b). 
(13) a. $\mathbf{S G}=\lambda \mathrm{P} \lambda \mathrm{x}[\mathrm{P}(\mathrm{x}) \wedge \mathbf{A t o ̂ m i c o}(\mathrm{x})]^{4}$

b. $\quad[[\mathbf{S G}(\mathrm{CN})]]=\{\mathrm{a}, \mathrm{b}, \mathrm{c}, \ldots\}$

(14) $\quad$ a. $\mathbf{P L}=\lambda \mathrm{P} \lambda \mathrm{x}[\mathrm{P}(\mathrm{x}) \wedge \operatorname{Molecular}(\mathrm{x})]$

b. $[[\mathbf{P L}(\mathrm{CN})]]=\{\{\mathrm{a}, \mathrm{b}\},\{\mathrm{a}, \mathrm{c}\}, \ldots,\{\mathrm{a}, \mathrm{b}, \mathrm{c}\} \ldots\}$

As abordagens tradicionais sobre a denotação dos NCs defendem que NCs contáveis denotam apenas indivíduos singulares e que plurais são operações sobre esses indivíduos singulares (cf. LINK, 1983; CHERCHIA, 1998). No entanto, vimos que SgNs e NCs no PB possuem átomos, pluralidades e partes em suas denotações. Consequentemente, não é correto postular, como tradicionalmente se faz, que denotações de NCs contáveis são sempre singulares em todas as línguas que fazem a distinção contável-massivo. Além disso, o fato de no PB o plural ser uma operação sobre a denotação neutra dos nomes comuns falsifica a tese de que o plural é sempre uma operação sobre singularidades em todas as línguas em que esta operação existe.

Vimos assim que a análise tradicional de que NCs contáveis denotam um conjunto de singularidades não se sustenta como hipótese geral para o comportamento das línguas humanas. Vimos também que a análise tradicional do plural enquanto uma operação que cria indivíduos plurais a partir de indivíduos singulares também não se sustenta para todas as línguas. Na próxima seção, veremos como diferentes teorias têm tratado da denotação dos nomes comuns e da expressão do número nas línguas naturais.

\section{A Distinção ContávEL-MASSIVO E SUA RELAÇÃo Com A EXPRESSÃo DO NÚMERO}

Nesta seção, investigaremos a relação entre a expressão de número e a distinção contável-massivo nas línguas naturais. Para isso, discutiremos as teorias de Link (1983), Chierchia (1998) e Borer (2005). Link (1983), assumindo que o aparato cognitivo humano vê o mundo como contendo tanto átomos quanto porções de matéria, propõe que a extensão de um nome contável é um conjunto de átomos, i.e., por singularidades, e que a extensão de tais $\backslash$ nomes no plural é um conjunto de pluralidades.

No que diz respeito aos nomes massivos, como, por exemplo, 'ouro', Link propõe que a extensão desses nomes é formada por um conjunto

\footnotetext{
[[Atomico a]] := 1 see [[a]] î A, onde A é o conjunto de entidades atômicas do

domínio.

do domínio (ver LINK, 1983).

[[Molecular a]] $:=1$ see $[[a]] \hat{I}^{+} \mathrm{A}$ onde $\mathrm{A}$ é o conjunto de entidades plurais
} 
contendo toda a matéria desse tipo e por subconjuntos contendo porções dessa matéria, que se dividem em novas partes sem que nunca átomos sejam obtidos. Assim, a extensão de 'ouro' conteria, para cada mundo, todo o ouro desse mundo e todas as porções que possam ser formadas com esse ouro. A extensão de nomes massivos, diferentemente da extensão dos nomes contáveis, é homogênea, ou seja, toda a vez que se subdivide uma porção de uma porção de ouro, o que se obtém é ainda ouro.

Assim, para Link (1983), nomes massivos possuem denotações distintas de nomes contáveis. Um nome massivo denota porções de matéria enquanto que um nome contável denota indivíduos singulares. Para este autor, o plural é uma operação sobre singularidades que resulta em um conjunto de pluralidades.

Chierchia (1998), por sua vez, assume que o aparato cognitivo humano vê o mundo como um conjunto contendo apenas entidades individuadas, sejam elas singulares ou plurais. Assim como Link (1983), propõe que a extensão de nomes contáveis é representada por um conjunto de singularidades e que a extensão de tais nomes no plural é representada por um conjunto de pluralidades. A parte mais controversa da proposta de Chierchia (1998) está relacionada aos nomes massivos. Para Chierchia a extensão de um nome massivo difere da extensão de um nome contável apenas porque contém tanto indivíduos singulares como indivíduos plurais. A denotação de um nome massivo seria então uma neutralização entre singular e plural, pois sua denotação inclui tanto indivíduos singulares como indivíduos plurais.

A concepção de Chierchia para os massivos pode ser ilustrada com o nome massivo 'mobília'. A denotação de 'mobília' inclui tanto sofás e poltronas individuais como conjuntos de, por exemplo, um sofá e duas poltronas. Essa concepção está ilustrada em (15). Já a concepção de Chierchia para a denotação dos nomes comuns contáveis singulares e plurais está ilustrada em (16) e (17).

(15) $[[$ mobília $]]=\left\{\right.$ sofá $_{1}$, sofá $_{2}, \ldots$, poltrona $_{1}$, poltrona $_{2}, \ldots$, mesa $_{1}, \ldots$, sofa $_{1}+$ poltrona $_{1}+$ poltrona $_{2}, \ldots .$, sofá $_{2}+$ mesa $\left._{4}+\ldots, \ldots\right\}$

(16) $\quad[$ sofád] $]=\left\{\right.$ sofá $_{1}$, sofá $_{2}$, sofá $_{3} \ldots$, sofá $\left._{n}, \ldots\right\}$

(17) $[[$ sofás $]]=\left\{\right.$ sofá $_{1}+$ sofáa $_{2}$, sofá $_{1}+$ sofá $_{3}, \ldots$, sofá $_{1}+$ sofá $_{2}+$ sofá $\left._{3}, \ldots\right\}$

Chierchia defende que tal teoria também se aplica a nomes comuns massivos como 'água', isso porque, para o autor, todos os nomes possuem átomos em sua extensão. A diferença seria que, em nomes como 'água', a noção do que seja um átomo de água varia de contexto para contexto sendo, portanto, mais vaga do que para outros nomes. Assim, para Chierchia (1998) o domínio de um nome massivo e de um nome contável é basicamente o 
mesmo e engloba tanto indivíduos atômicos quanto indivíduos plurais.

A proposta de Borer (2005) implica que o aparato cognitivo humano vê o mundo como basicamente massivo. Para a autora, todos os nomes são originalmente massivos no sentido de que denotam matéria não individuada. Ela defende que a distinção contável-massivo é uma distinção obtida através da sintaxe. Um núcleo funcional - DIV(ider) - transforma um nome massivo em contável sem fornecer, no entanto, qualquer unidade de contagem. Segundo a autora, singular, plural e classificadores são instanciações de DIV e realizam operações sobre denotações massivas e essas operações fornecem divisões e não unidades pré-determinadas.

Nesta seção, vimos três teorias sobre a denotação dos nomes comuns. A primeira, representada por Link (1983), postula que existem dois tipos de denotações - individuadas e não-individuadas. A segunda afirma que todos os NCs têm denotações individuadas e é representada por Chierchia (1998). A terceira postula que todos os NCs possuem denotações massivas e é defendida por Borer (2005). Vimos também como cada uma delas define o papel semântico da morfologia de número. o SgN no PB.

Na próxima seção avaliaremos essas teorias frente aos dados sobre

\section{O PB E As Teorias Sobre a Denotação dos Nomes Comuns E SOBRE A EXPRESSÃo DO NÚMERO}

Nesta seção, trataremos de discutir os dados sobre o SgN no PB frente às teorias apresentadas na seção anterior.

Levando em consideração o $\mathrm{PB}$, podemos observar que se a teoria de Chierchia estiver certa quando propõe que ser massivo é o mesmo que ser neutro para número, todos NCs e SgNs deveriam ser considerados massivos no PB. Vimos na seção 1 que a denotação de um nome comum como, por exemplo, 'maçã' em (18), é neutra para número, sendo a verdade da sentença indiferente ao fato de terem sido compradas uma ou mais maçãs ou mesmo ao fato de se tratarem de porções ou fatias de maçã. Uma denotação desse tipo seria semelhante à proposta por Chierchia para os nomes massivos (ver (15)) e está ilustrada em (19).

(18) João comprou maçã.

(19) $[[$ maçã $]]=\left\{\right.$ maça $\tilde{a}_{1}$, maça $\tilde{a}_{2}$, maça $\tilde{a}_{3}, \ldots$, maça $\tilde{a}_{1}+$ maça $_{2}, \ldots$, maçã ${ }_{1}+$ maçã $_{2}+$ maça $\left._{3}, \ldots\right\}$

Outra evidência que poderia nos levar a achar que os NCs e SNs no PB são massivos é o fato de que SgNs podem co-ocorrer com 
quantificadores massivos, como 'muito' e com 'suficiente', como pode ser observado em (20) e (21).

(20) Tem muita mala no carro.

(21) Eu escrevi carta suficiente por hoje.

No entanto, há diferenças morfossintáticas entre nomes que são ontologicamente massivos, como 'ouro' ou 'água', e nomes que são ontologicamente contáveis como 'maçã'. Em (22), o sintagma que contém a palavra 'ouro' precisa de um classificador ou de um sintagma de medida, seja ele explícito ou fornecido pelo contexto, para que possa ser interpretados. O mesmo não acontece com 'maçã' em (23) que é diretamente interpretável. Isso nos leva a concluir que Link (1983) está provavelmente certo quando estabelece domínios diferentes para nomes contáveis e massivos.

(22) *dois ouros.

(23) duas maçã (s)

Outra evidência que confirma a tese de Link (1983) é o fato de que o plural de um nome contável pode indicar pluralidade de indivíduos ou de tipos, mas o plural de um nome massivo indica apenas pluralidade de tipos ou a existência de algum classificador implícito no contexto (cf. CHUNG, 2000). Na sentença (24a), como já vimos, afirma-se que foram compradas uma ou mais maçãs ou partes de maçã. Já (24b) é ambígua entre afirmar que foram compradas mais de uma maçã ou mais de um tipo de maçã. A sentença (25a), com o SgN massivo 'óleo', afirma que foi comprada uma quantidade indeterminada de óleo. Já a sentença $(25 \mathrm{~b})$ não possui o mesmo tipo de ambiguidade de (24b). Na ausência de um sintagma de medida fornecido pelo contexto, ela significa que foram comprados mais de um tipo de óleo.
(24) a. Eu comprei maçã.
b. Eu comprei maçãs.
(25) a. Eu comprei óleo.
b. Eu comprei óleos.

Schmitt e Munn (1999) observam também que nomes massivos são incompatíveis com predicados que requerem atomização, enquanto que nomes contáveis são compatíveis com estes predicados. Em (26-27), os predicados 'cai/anda um atrás do outro' só formarão sentenças gramaticais se saturados por um nome contável como 'elefante' em (26). 'Ouro', como é um nome massivo, não é capaz de tornar a sentença (26) gramatical. Os 
autores observaram também que pronomes reflexivos e recíprocos são compatíveis com nomes contáveis, mas não com nomes massivos. A diferença é ilustrada pelo contraste entre a gramaticalidade de (28) e a agramaticalidade de (29).

(26) Elefante anda um atrás do outro.

(27) *Ouro cai um atrás do outro.

(28) Criança briga uma com a outra.

(29) *Ouro realça um o outro.

Desse modo, podemos concluir sobre o PB que há uma distinção lexical entre nomes contáveis e massivos. Pelo menos nesta língua, NCs não são todos lexicalmente massivos. Os nomes comuns já vêm marcados no léxico como massivos ou contáveis. Assim, concluímos também que, pelo menos para o PB, o papel da morfologia de número não tem, como prevê Borer (2005), o papel de criar a contabilidade, de efetivar divisões em denotações massivas. Além disso, ficou claro também que nomes com denotações massivas e nomes com denotações contáveis neutras para número comportam-se de modo distinto. Consequentemente, uma denotação massiva não é apenas uma denotação neutra para número, como defendido por Chierchia (1998). Ser massivo é denotar matéria indiferenciada e ser contável é possuir entidades individualizadas sob sua denotação (LINK, 1983).

$\mathrm{Na}$ próxima seção veremos que as propostas de Chierchia e de Borer não são postas em questão apenas pelo comportamento do $\mathrm{PB}$, mas também por outras línguas de famílias variadas.

\section{VARIAÇÃo TRANSLINGUística NA EXPRESSÃo Do NÚMERo E SUA RELAÇÃo COM A DisTINÇÃo MASSIVO-CONTÁvEL}

Nesta seção investigaremos empiricamente a relação entre a marcação de número, o uso de classificadores e a distinção contável-massivo nas línguas naturais.

Em algumas línguas, para se expressar a cardinalidade de um conjunto, não basta juntar um numeral a um nome, pois o uso de classificadores é obrigatório. Por esse motivo, muitos autores têm proposto que o uso de classificadores é necessário porque cria indivíduos a partir de denotações originalmente massivas (cf. GIL, 1987; CHIERCHIA, 1998; BORER, 2005). Elas não apresentam flexão de plural ou pelo menos, não apresentam uma flexão de plural como a conhecida nas línguas indo-europeias. $O$ chinês é um exemplo desse tipo de língua. O contraste entre (30a) e (30b) mostra que, como em todas as línguas, um nome massivo precisa ser classificado 
para poder ser contado. Já o contraste entre (31a) e (31b) diferencia o chinês de outras línguas como o PB, pois em chinês não se pode contar nomes ontologicamente contáveis, como 'caneta', sem a presença de um classificador. $^{5}$

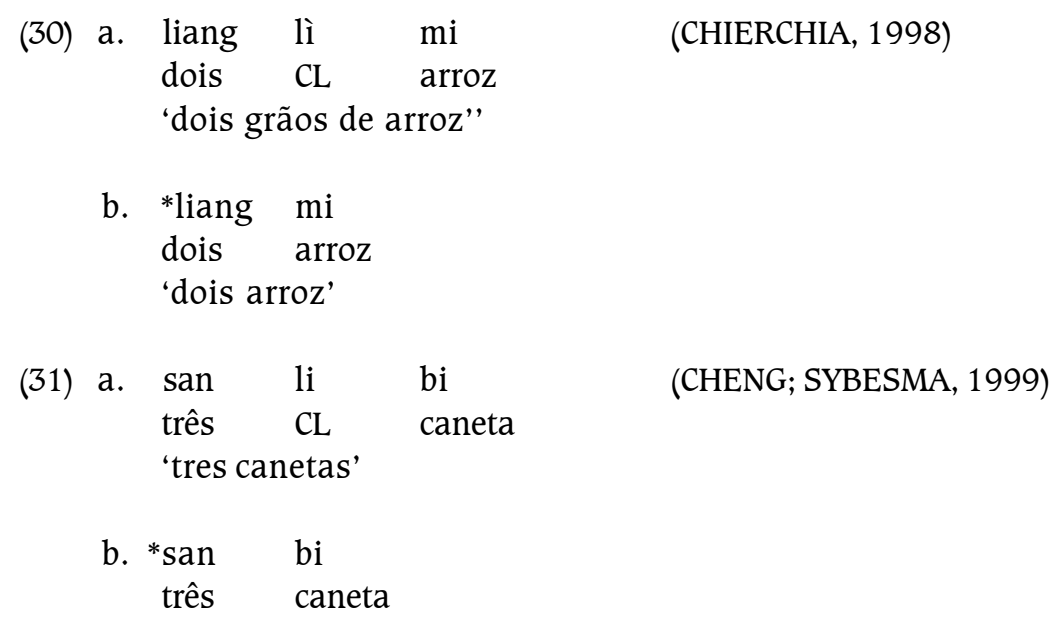

Segundo Gil (1987), Chierchia (1998) e Borer (2005), existiria uma distribuição complementar entre a ocorrência de classificadores e a flexão de plural nas línguas naturais. Assim, línguas que possuem morfologia de número não possuiriam classificadores e línguas classificadoras não possuiriam morfologia de número. Para Chierchia e Gil, a distribuição complementar ocorre porque a morfologia de número só é possível em línguas que possuem a distinção contável-massivo, já que só é possível pluralizar nomes contáveis. Por outro lado, línguas classificadoras e sem morfologia de número teriam apenas nomes massivos e, por isso, necessitariam de classificadores para criar indivíduos passíveis de serem contados. Já para Borer, a complementariedade acontece porque tanto a morfologia de número como os classificadores cumprem o mesmo papel de criarem divisões em denotações universalmente massivas. Para a autora, uma língua pode até possuir tanto morfologia de número como classificadores, mas ambas as operações nunca poderiam co-ocorrer.

No entanto, a hipótese da complementariedade tem sido empiricamente contestada devido a fatos como que discutimos a seguir. Existem línguas, por exemplo, que possuem tanto um sistema de classificadores quanto flexão de número (cf. AVELAR, 2005; BORER 2005).

5 Abreviaturas utilizadas: CL-classificador, SG-singular, DL-dual, PL-plural, DECL-declarativo, NFUT-não futuro, PART-particípio, aux-auxiliar, redupl-reduplicação, Qquantificador. 
o Yágua é um exemplo desse tipo de língua. A língua possui morfologia de número - singular, dual e plural - como se pode ver em (32a-c). Possui também classificadores que devem ser obrigatoriamente usados na presença de numerais, como ilustra (33).

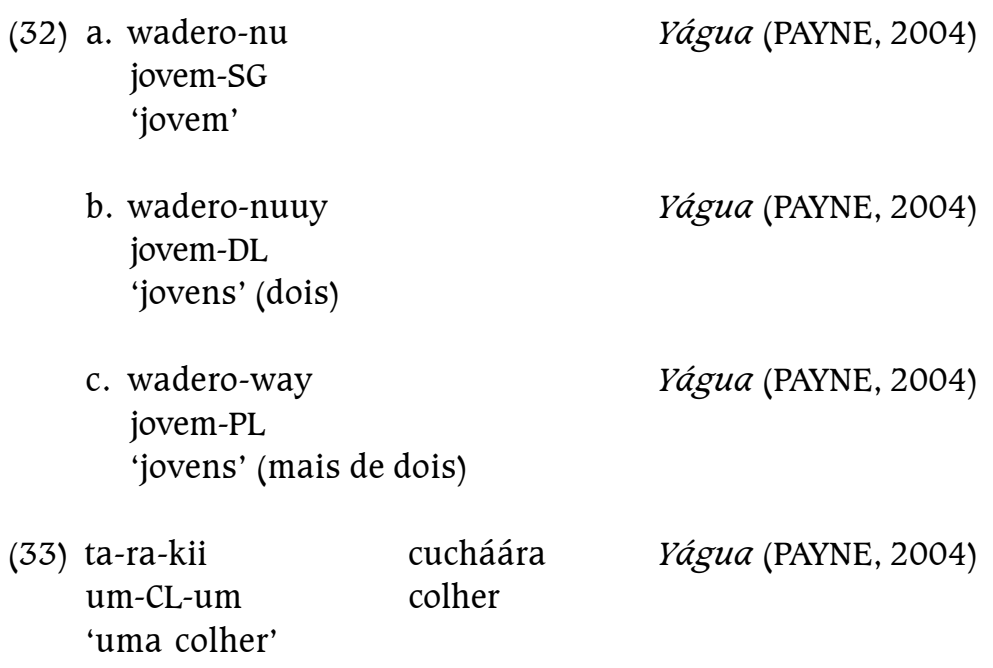

Por outro lado, existem línguas como o Dëne Suliné e o Karitiana que não possuem nem sistema de classificação nem flexão de número (cf. WILHELM, 2005, MÜLLER et al., 2006). Em Karitiana, as expressões nominais ocorrem nuas, sem marcação de número ou de definitude, sendo que os nominais nus nessa língua são neutros em relação ao número que expressam, como se pode ver pela multiplicidade de traduções possíveis para a sentença (34). E, em contextos de contagem, não há ocorrência de classificadores ou de morfologia de número, como ilustrado em (35) e (36).

(34) Taso naka'yt boroja Karitiana (MÜLLER et al., 2006) taso naka-'y-t boroja homem DECL-comer-NFUT cobra 'Um/o(s)/algum(s) homem(s) comeu(eram) (uma/a(s)/alguma(s)) cobra(s)'

(35) Myhint 'ejepo naakat i'ot. Karitiana myhint 'ejepo na-aka-t i-'ot- $\varnothing$ (MÜLLER et al.,2006) uma pedra DECL-aux-NFUT PART-cair-NFUT 'Uma pedra caiu' 
(36) Sympomp 'ejepo naakat i'orot. Karitiana sypomp 'ejepo na-aka-t i-'ot-<0>t (MÜLLER et al., 2006) duas pedra DECL-auc-NFUT PART-cair-redupl-NFUT 'Duas pedras caíram'

Se classificadores são necessários para criar indivíduos em denotações inerentemente massivas, esperaríamos que, em contextos de quantificação que implicam em individuação, eles estivessem obrigatoriamente presentes nas línguas classificadoras. No entanto, isso não é verdadeiro (GIL; TSOULAS, 2005). Em japonês e em coreano, por exemplo, sintagmas com quantificados com leitura universal distributiva ocorrem necessariamente sem classificadores, como ilustrado em (37) para o japonês.

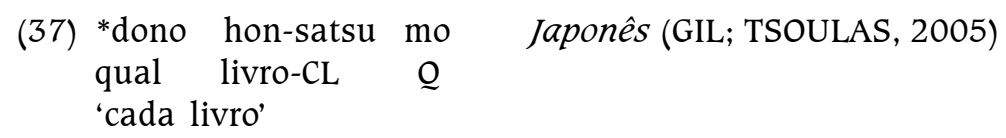

Apesar de Gil (1987) e Chierchia (1998) afirmarem que as denotações dos NCs em línguas classificadoras são sempre massivas, essa tese não se sustenta. A distinção contável-massivo está presente tanto em línguas classificadoras como em línguas não classificadoras (cf. CHENG; SYBESMA, 1999; DOETJES, 1979 para o chinês, WILHELM, 2005 para o dëne suliné, MÜLLER et al., 2006 para o Karitiana). Em Karitiana, alguns nomes podem ser diretamente contados, como podemos observar em (38) e (39) e outros não, como em (40).
(38) Myhint 'ejepo naakat i'ot. Karitiana
Myhint 'ejepo na-aka-t i-'ot- $\varnothing$
Uma pedra DECL-aux- NFUT part-cair-NFUT
'Uma pedra caiu'

(39) Sympomp ejepo naakat i'orot. Karitiana

sypomp 'ejepo na-aka-t i-'ot-<0>t (MÜLLER et al., 2006)

duas pedra DECL-aux-NFUT part-cair-redupl- NFUT

'Duas pedras caíram'

(40) *Myhint ouro naakat i'ot. Karitiana

myhint ouro na-aka-t i-'ot- $\varnothing$ (MÜLLER et al., 2006)

um ouro DECL-aux- NFUT part-cair- NFUT

'Um ouro caiu' 
Doetjes (1997) apresenta como uma das evidências a favor da existência da distinção contável-massivo no chinês o fato de que o classificador geral ge ('unidade') só é possível com nomes contáveis como podemos ver contrastando (41) com (42). Em (41), temos o classificador ge usado com o nome contável shu ('livro') e o sintagma é gramatical. Já em (42), temos o mesmo classificador usado com o nome massivo rou ('carne') e o sintagma é agramatical. Esses comportamentos põem em questão a existência de uma correlação necessária entre a presença de classificadores e a presença de uma denotação massiva.

$$
\begin{aligned}
& \text { (41) san ge shu } \\
& \text { três CL livro } \\
& \text { 'três (unidades de) livros' } \\
& \text { (42) *san ge rou } \\
& \text { três CL carne } \\
& \text { 'três (unidades de) carne' }
\end{aligned}
$$

Em síntese, podemos afirmar que os nomes comuns podem ser diretamente contados sem precisarem necessariamente de flexão de número ou de classificadores, exceto quando se tratam de nomes massivos. Isto implica que uma denotação neutra para número não exclui contabilidade ou individuação e que a morfologia de número e os classificadores não são necessariamente divisores ou pluralizadores, ao contrário do que defendem Borer (2005) e Gil (1987) e Chierchia (1998).

\section{CONCLUSÃO}

Com base no que vimos acima, podemos afirmar que muitas das ideias sobre a distinção contável-massivo e a expressão do número nas línguas naturais estão equivocadas. Primeiramente, vimos que a denotação de NCs contáveis não é necessariamente uma denotação composta apenas de indivíduos singulares. Vimos também que uma denotação neutra para número não é necessariamente uma denotação massiva (cf. PARAGUASSU, 2005 para PB, DOETJES, 1997 para o chinês) e que a morfologia de número e os classificadores não são necessariamente operações de divisão sobre a denotação de nomes comuns massivos ou criadores de indivíduos plurais a partir de indivíduos singulares.

Assim, fechamos o artigo levantando a possibilidade de que a distinção contável-massivo seja um universal semântico. Outro universal possível e já postulado por Kratzer (2003) é o de que todos os predicados 
nas línguas naturais são em princípio cumulativos. i.e., neutros para diferenças de número. Ou seja, uma denotação neutra para número seria geral para todos os nomes contáveis.

\title{
RESUMO
}

Este artigo investiga a expressão de número e da distinção contável-massivo no sistema nominal das línguas naturais. Em particular, discute o comportamento do nominal nu no Português Brasileiro com relação à expressão da distinção de número e da distinção contável-massivo. O objetivo é tentar responder às seguintes questões: (i) Quais são as denotações possíveis para os nomes comuns nas línguas naturais? (ii) Qual seria o papel semântico da morfologia de número e dos classificadores? (iii) O que é ser um nome massivo ou contável? Defendemos que a denotação de nomes comuns contáveis não é necessariamente uma denotação composta apenas de indivíduos singulares e que uma denotação neutra para número não é necessariamente uma denotação massiva. Além disso, mostramos que a morfologia de número e os classificadores não são necessariamente operações de divisão sobre a denotação de nomes comuns massivos ou criadores de indivíduos plurais a partir de indivíduos singulares.

Palavras-chave: Nomes Comuns; Distinção Contável-Massivo; Número.

\begin{abstract}
This paper investigates the expression of number and of the count-mass distinction in natural languages. In particular, it deals with the behavior of bare singulars in Brazilian Portuguese. The goal of the paper is to answer to the following questions: (i) What are possible denotations for common nouns? (ii) What is the role of classifiers and of number morphology? (iii) What is it to be mass or count? We claim that denotations of common nouns aren't necessarily composed of singular individuals. We also claim that number-neutral denotations are not equivalent to mass denotations. Besides that, we show that number morphology and classifiers are not necessarily dividing operations on the denotation of mass nouns; nor creators of plural individuals out of singular ones.
\end{abstract}

Keywords: Common Nouns; Count-Mass Distinction; Number. 


\section{REFERÊNCIAS}

AVELAR, M. Relatório Científico FAPESP, ms. 2005.

BORER, H. Structuring Sense. Oxford: Oxford University Press, 2005

CHENG, L. L; SYBESMA, R. Bare and not-so-bare nouns and the structure of NP. Linguistic Inquiry, v. 30, n. 4, p. 509-542,1999.

CHIERCHIA, G. Reference to kinds across languages. Natural Language Semantics, v. 6, p. 339-405, 1998.

DOETJES. Quantifiers and Selection. On the Distribution of Quantifying Expressions in French, Dutch and English. Ph.D dissertation, Leiden University, 1997.

CHUNG, S. On Reference to Kinds in Indonesian. Natural Language Semantics, v. 8, n. 2, p. 157-171, 2000.

GIL, D. Definiteness, noun phrase configurationality, and the count-mass distinction. In: REULAND, E. J; MEULEN, A. G .B. ter. (Eds.). The Representation of (In)definiteness. Cambridge, MA: MIT Press, 1987.

GIL, K.-H; TSOULAS, G.. Quantification, the Nominal Mapping Parameter, and DP Structure in Korean and Japanese. Talk presented at QP Structure, Nominalizations, and the Role of DP, 2005.

KRATZER, A. The Event Argument and the Semantics of Verbs. Ch3. 2003. Disponível em: $<$ http://semanticsarchive.net/Archive/GU1NWM4Z>. Acesso em: 11/07/2009.

LINK, G.. The logical analysis of plurals and mass terms: a lattice theoretical approach. In: BÄUERLE, R.; SCHWARZE, C.; STECHOW, von A. (Eds.). Meaning, Use and Interpretation of Language. Berlin: de Gruyter, 1983. p. 303-323.

LONGOBARDI, G. Reference to Kinds and Proper Names: a Theory of N movement in Syntax and Logical Form. Linguistic Inquiry, v. 25, n. 4, p. 609-665, 1994.

MÜLLER, A. Genericity and the denotation of common nouns in Brazilian Portuguese. In: WEERLE, A; KIM, J-Y. (Eds.). The Semantics of Under-Represented Languages in the Americas, UMOP, 25. Amherst, MA: GLSA, The University of Massachusetts, 2001. p. $72-$ 80 .

MÜLLER, A.; STORTO, L; COUTINHO-SILVA. Number and the Count-Mass Distinction in Karitiana. Revista da ABRALIN, 2006. No prelo.

PARAGUASSU, N. A Distinção Massivo-Contável no sistema nominal. Dissertação (Mestrado) - Universidade de São Paulo, 2005.

PAYNE, D. The Source and Function of Yagua Classifiers, ms. 2004.

PELLETIER, F. Mass terms: some philosophical problems. Dordrecht: Reidel, 1979.

SCHMITT, C; MUNN, A.. Against the Nominal Mapping Parameter: Bare Nouns in Brazilian Portuguese. NELS, v. 29,1999.

WILHELM, A. Bare Nouns in Dëne Suliné. Talk presented at SULA-3, Buffalo, USA, 2005.

Submetido em: 17/03/2007.

Aceito em: 25/09/2007 